\title{
Caracterização física de café após torrefação e moagem
}

\section{Physical characterization of coffee after roasting and grinding}

\author{
Gabriel Henrique Horta de Oliveira ${ }^{1 *}$; Paulo Cesar Corrêa ${ }^{2}$; \\ Fábio Lúcio Santos²; Wander Luiz Vasconcelos ${ }^{3}$; Carlito Calil Júnior ${ }^{4}$; \\ Fernanda Machado Baptestini ${ }^{5}$; Guillermo Asdrúbal Vargas-Elías ${ }^{5}$
}

\section{Resumo}

A escoabilidade é uma importante característica do manuseio para a eficiência e a confiabilidade das operações pós-colheita, sendo regida pelas propriedades físicas do produto. A determinação das propriedades físicas é um importante fator na formulação de projetos de maquinários e dimensionamento de operações pós-colheita, podendo impactar consideravelmente na qualidade do produto, influenciando diretamente o custo da operação e o lucro da empresa. Assim, objetivou-se avaliar e determinar as propriedades físicas: ângulo de repouso, massa específica unitária e aparente, porosidade e as coordenadas de cor, análise da granulometria, teor de água, atividade de água, ângulo de atrito interno e efetivo ângulo de atrito interno do café, bem como avaliar a influência de diferentes níveis de torra e granulometria sobre essas propriedades. Foram utilizados grãos de café cru (Coffea canephora e Coffea arabica), descascados e secos, e torrados em dois níveis: média clara (SCAA\#65) e moderadamente escura (SCAA\#45). Após a torrefação, os grãos foram moídos em três granulometrias: fina $(0,59 \mathrm{~mm})$, média $(0,84 \mathrm{~mm})$ e grossa $(1,19 \mathrm{~mm})$, segundo normas de classificação da ABIC, além de mantido o lote de café inteiro. A granulometria e o nível de torrefação afetaram significativamente as propriedades físicas do café. As amostras de café torradas ao nível moderadamente escuro obtiveram menores valores de teor de água, atividade de água, ângulo de repouso, massa específica unitária e aparente. Já os cafés de granulometria mais fina apresentaram aumento de ângulo de atrito interno e efetivo ângulo de atrito interno, ângulo de repouso, massa específica unitária e aparente, porosidade e decréscimo de atividade de água.

Palavras-chave: Propriedades físicas, pós-colheita, escoabilidade

\begin{abstract}
Flowability is an important characteristic of handling process for efficiency and reliability purpose of post-harvest operations, which are governed by the physical properties of the product. Physical properties determination is an important factor for formulation of machinery projects and sizing of post-harvest operations, which may impact considerably on the products quality, influencing directly the operation cost and company profit. Thus, the objective of this work was to evaluate and determinate some physical properties (repose angle, unit and bulk density, porosity and color coordinates, particle size, moisture content, water activity, angle of internal friction and effective angle of internal friction)
\end{abstract}

${ }^{1}$ Prof. Dr. em Engenharia Agrícola, Instituto Federal de Brasília, IFB, Campus Gama, Brasília, DF. E-mail: gabriel.oliveira@ifb. edu.br

2 Profs. Drs. do Dept ${ }^{\circ}$ de Engenharia Agrícola, Universidade Federal de Viçosa, UFV, Viçosa, MG. E-mail: copace@ufv.br; fabio. 1s@ufv.br

${ }^{3}$ Prof. Dr. do Dept ${ }^{\circ}$ de Engenharia Metalúrgica e de Materiais, Universidade Federal de Minas Gerais, UFMG, Belo Horizonte, MG.E-mail: wlv@demet.ufmg.br

${ }^{4}$ Prof. Dr. do Dept ${ }^{\circ}$ de Engenharia de Estruturas, Universidade de São Paulo, USP, São Carlos, SP. E-mail: calil@sc.usp.br

${ }^{5}$ Discentes de Doutorado em Engenharia Agrícola, UFV, Viçosa, MG. E-mail: fbaptestini@yahoo.com.br; guilhermo.elias@ufv.br

* Autor para correspondência 
of coffee, such as to evaluate the influence of different roast and grinding degrees over these properties. Crude grain coffee (Coffea canephora and Coffea arabica), dehulled and dried were used. These were roasted at two levels: medium light and medium-dark brown, which Agtron numbers are, respectively, SCAA\#65 and SCAA\#45. After roasting process, grains were grinded at three particle sizes (thin, medium and thick). Both particle size and roast degree significantly affected physical properties of coffee. Coffee samples roasted at medium dark level obtained lower values of moisture content, water activity, repose angle, bulk and real density. Coffee samples grinded at level thin presented an increase of angle of internal friction and effective angle of internal friction, repose angle, bulk and real density, porosity and decrease of water activity values.

Key words: Physical properties, post-harvest, flowability

\section{Introdução}

A demanda crescente de cafés pelos mercados importadores, tanto quantitativamente como qualitativamente, bem como a concorrência de outros países produtores acabam por incentivar a indústria nacional cafeeira a melhorar seus produtos e processos. O constante manuseio do café torrado, tanto o grão inteiro como o moído, é uma parte importante desses processos. O manuseio de materiais é um importante fator na operação de qualquer indústria alimentícia e pode afetar consideravelmente a qualidade do produto, influenciando diretamente o custo da operação e o lucro da empresa (ROBBERTS, 2002). A cada movimentação dos produtos, o custo final sofre adições, além de aumentar o risco de danos aos produtos e de acidentes podendo alcançar até 50 $\%$ do valor total da manufatura do produto final (ROBBERTS, 2002).

No caso de café industrializado, a escoabilidade é uma importante característica do manuseio para a eficiência e a confiabilidade da operação e é muito influenciada pelas propriedades físicas do produto. Projetos de máquinas para o processamento, classificação e dimensionamento de outros equipamentos destinados à pós-colheita de produtos agrícolas requerem dados relativos às propriedades físicas. Assim, obter informações quanto às características destes produtos é importante para o correto manuseio e processamento dos mesmos (SCHUBERT, 1987), diminuindo assim os custos. O estudo das propriedades físicas ainda possibilita a predição do comportamento dos produtos agrícolas relativo às respostas de tratamento físicos e químicos, de forma a permitir a manutenção da qualidade e segurança dos alimentos processados (WILHELM; SUTER; BRUSEWITZ, 2004), garantindo assim a comercialização do produto com menor depreciação da qualidade.

Segundo Robberts (2002), o adequado manuseio e transporte de materiais proporcionam: diminuição do custo operacional por meio do correto uso da força de trabalho, equipamentos e espaço; diminuição do tempo de processamento; movimentação ininterrupta dos materiais; prevenção de injúrias e acidentes devido ao manuseio incorreto dos produtos; produto de melhor qualidade, e; redução da perda dos produtos. Sendo assim, informações que visam aperfeiçoar e/ou desenvolver novos equipamentos e métodos para o manuseio de produtos possibilitam a diminuição do trabalho, das perdas e, por conseguinte, do custo do material final do produto em menor tempo e com máxima eficiência e segurança. Dessa forma, o estudo das propriedades físicas de café é necessário para a indústria cafeeira brasileira.

Diante do exposto, objetivou-se com este trabalho avaliar e determinar as propriedades físicas: ângulo de repouso, massa específica unitária e aparente, porosidade e as coordenadas de cor, análise da granulometria, teor de água, atividade de água, ângulo de atrito interno e efetivo ângulo de atrito interno do café, bem como avaliar a influência de diferentes níveis de torra e granulometria sobre essas propriedades. 


\section{Material e Métodos}

Foram utilizados grãos de café cru (Coffea canephora e Coffea arabica), descascados e secos, adquiridos de comércio regional da Zona da Mata de Minas Gerais. Os grãos foram submetidos a uma triagem para eliminar os grãos deteriorados, danificados e brocados, a fim de se obter uma matériaprima homogênea e com o mínimo de defeitos. Grãos provenientes de frutos de bica corrida foram utilizados. O teor de água médio inicial dos grãos foi de $12,61 \%$ (base seca), tendo sido determinado pelo método gravimétrico, utilizando uma estufa com circulação forçada de ar a $105 \pm 1{ }^{\circ} \mathrm{C}$ por $24 \mathrm{~h}$ (BRASIL, 2009), em triplicata.

Os grãos de café de ambas as espécies, após a triagem, foram encaminhados para o processo de torrefação, na qual foi utilizado um torrefador de queima direta de gás GLP, com cilindro em movimento rotativo a $45 \mathrm{rpm}$, com pré-aquecimento e capacidade de 350 g de café cru. O nível de cada torrefação dos grãos de café foi identificado por meio do acompanhamento e comparação da cor das amostras com o número padrão de cor Agtron, assessorado por um profissional treinado. Dois níveis de torra foram obtidos: média clara (MC) e moderadamente escura (ME), cujos números Agtron correspondentes são, respectivamente, SCAA\#65 e SCAA\#45 (Figura 1). Para garantir a uniformidade das torras, foi utilizado um termômetro infravermelho, marca Mult-Tempo portátil que fornece leituras entre -50 e $500{ }^{\circ} \mathrm{C}$, com tempo de resposta de $1 \mathrm{~s}$ e resolução de $0,1^{\circ} \mathrm{C}$. Ao se atingir a temperatura do torrefador de $285^{\circ} \mathrm{C}$, os grãos perderam, em média, 15,85 e 18,74 gramas de massa, respectivamente para as torras MC e ME (VARGAS-ELÍAS, 2011). Ao atingir os dois níveis de torra mencionados, o produto foi retirado do torrefador e imediatamente esfriado utilizando-se ar ambiente.

Figura 1. Níveis de torra empregados: média clara (A) e moderadamente escura (B).

(A)

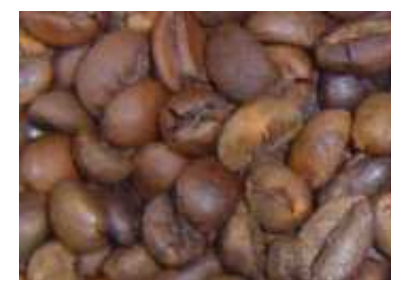

(B)

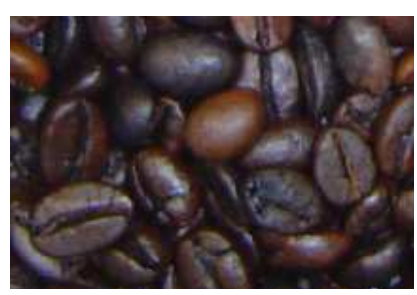

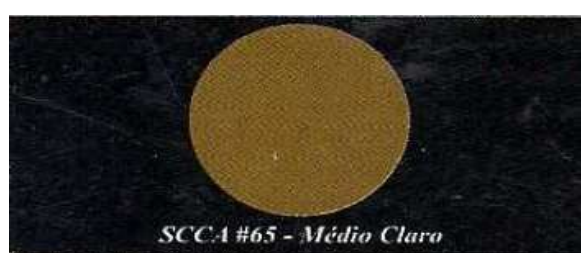

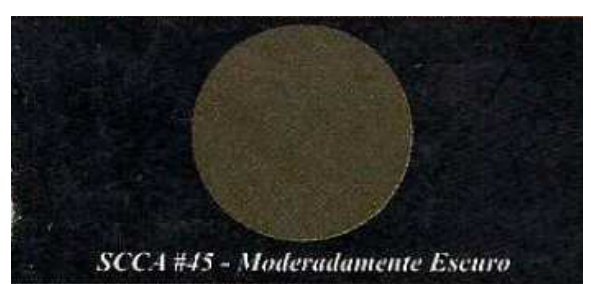

Fonte: Vargas-Elías (2011). Adaptado.

Após o processo de torrefação, os grãos foram processados em moinho Mahlkönig em três granulometrias diferentes, conforme recomendações da ABIC (2013) (Tabela 1): fina $(0,59 \mathrm{~mm})$, média $(0,84 \mathrm{~mm})$ e grossa $(1,19 \mathrm{~mm})$, além de mantido o lote de café inteiro. As amostras preparadas foram analisadas quanto a diferentes propriedades físicas, descritas a seguir.

O aparelho Aqualab 4 TE, da Decagon Devices, foi utilizado para a determinação da atividade de água $\left(\mathrm{a}_{\mathrm{w}}\right)$ de café torrado inteiro e moído. Este 
equipamento fornece leituras de atividade de água no intervalo de 0,050 a 1,000 (decimal), com precisão de $\pm 0,003$ e resolução de 0,0001. Cada amostra foi avaliada em triplicata e a média das três determinações foi utilizada.

A metodologia para a obtenção do ângulo de repouso seguiu a proposta por Silva et al. (2006), em que utilizou-se uma plataforma cilíndrica circular de diâmetro de $20 \mathrm{~cm}$, contendo, em seu centro, uma haste graduada onde o produto foi depositado, proveniente de um funil de recepção de amostra de grãos a uma altura de $0,60 \mathrm{~m}$. Em seguida, mediuse a altura do talude formado pelo produto e a plataforma. De posse destas informações, o ângulo de repouso foi obtido por meio do arco tangente de um triângulo formado pela base do produto e a altura do talude. Cada amostra foi avaliada em triplicata e a média das três determinações foi utilizada.

Tabela 1. Classificação com base na percentagem de retenção em peneiras granulométricas números 12, 16, 20, 30 e fundo, em equipamento específico com agitação por 10 minutos e reostato na posição 5, ou similar.

\begin{tabular}{cccccc}
\hline \multirow{2}{*}{ Moagem } & \multicolumn{3}{c}{ \% de retenção } & \multicolumn{2}{c}{ Tolerância para a \% que passa da peneira 30 } \\
\cline { 2 - 6 } & Peneiras 12 e 16 & Peneiras 20 e 30 & Fundo & Mínimo & Máximo \\
\hline Grossa & 33 & 55 & 12 & 9 & 15 \\
Média & 7 & 73 & 20 & 16 & 24 \\
Fina & 0 & 90 & 30 & 25 & 40 \\
\hline
\end{tabular}

Fonte: ABIC (2013).

A massa específica unitária $\left(\rho_{u}\right)$ ou real foi determinada com o uso de um picnômetro a hélio, a partir das Equações 1 e 2, em cinco repetições. $\mathrm{O}$ picnômetro a hélio utilizado foi o Multipycnometer modelo MVP-4DC (Quantachrome Corporation, EUA), cuja operação é realizada conforme o método de deslocamento de gás.

$$
\begin{gathered}
P_{1}\left(V_{a}-V_{s}\right)=P_{2}\left(V_{a}-V_{s}+V_{e}\right) \\
\rho_{u}=\frac{m_{g}}{V_{s}}
\end{gathered}
$$

em que, $\mathrm{P}_{1}=$ pressão inicial, $\mathrm{Pa} ; \mathrm{P}_{2}=$ pressão final, $\mathrm{Pa} ; \mathrm{V}_{\mathrm{a}}=$ volume da câmara da amostra, $\mathrm{m}^{3} ; \mathrm{V}_{\mathrm{s}}=$ volume do sólido (café), $\mathrm{m}^{3} ; \mathrm{V}_{\mathrm{e}}=$ volume da câmara de expansão, $\mathrm{m}^{3} ; \rho_{\mathrm{u}}=$ massa específica unitária, $\mathrm{kg}$ $\mathrm{m}^{-3} ;$ e $\mathrm{m}_{\mathrm{g}}=$ massa de café torrado, $\mathrm{kg}$.

A massa específica aparente $\left(\rho_{\mathrm{ap}}\right)$ foi determinada com uma balança de peso hectolitro do tipo Burrows com capacidade de $1 \mathrm{~L}$, em chapa de aço, em cinco repetições. A porosidade $(\varepsilon)$ foi determinada indiretamente de acordo com a Equação 3
(MOHSENIN, 1986), tradicionalmente utilizada para produtos agrícolas.

$$
\varepsilon=\left(1-\frac{\rho_{a p}}{\rho_{u}}\right) \times 100
$$

em que, $\varepsilon=$ porosidade, $\%$; e $\rho_{\text {ap }}=$ massa específica aparente, $\mathrm{kg} \mathrm{m}^{-3}$.

A cor foi determinada empregando um colorímetro (MiniScan XE Plus 45/0-L, CIELAB, Reston, Estados Unidos), em três repetições, com leitura direta de reflectância das coordenadas $\mathrm{L}^{*}$ (luminosidade), a* (componente vermelho/verde) e b* (componente amarelo/azul), e iluminante D65. A partir dos valores de $L^{*}, a^{*}$ e b*, foram calculados o croma $\left(\mathrm{C}^{*}\right)$ e o ângulo hue $\left(\mathrm{h}^{*}\right)$.

A análise granulométrica consiste na determinação das dimensões das partículas que constituem as amostras de café torrados e moídos. Os grãos de café inteiro não foram avaliados, uma vez que as medidas são realizadas em solução líquida com água destilada, realizando-se a análise com o sólido em suspensão. 
A granulometria foi avaliada com o uso de granulômetro Cilas, modelo 1064, em três repetições. Este equipamento fornece a distribuição granulométrica na faixa entre 0,04 a $500 \mu \mathrm{m}$.

A determinação do ângulo de atrito interno (Фi) e efetivo ângulo de atrito interno (Фe) foi realizada em triplicata, utilizando-se um equipamento de cisalhamento modelo TSG 70-140, construído com base no aparelho de cisalhamento de Jenike. A metodologia empregada foi a proposta por Milani (1993), sendo que, para a execução do ensaio, foram utilizadas as recomendações da norma inglesa - BMHB (1985), Jenike e Johanson (1979) e o Manual de Operação da Máquina TSG 70-140.

O delineamento inteiramente casualizado foi empregado neste trabalho, com suas repetições variando de acordo com a propriedade física analisada. $O$ trabalho foi realizado em dois experimentos: um tendo como matéria-prima grãos de café da espécie Coffea arabica L. e o outro utilizando grãos de café da espécie Coffea canephora Pierre. Para cada experimento, os dados foram avaliados por meio de análise de variância, comparando-se as médias por meio do teste de Tukey, adotando-se o nível de $5 \%$ de probabilidade. A análise estatística foi realizada utilizando-se do programa $\mathrm{SAEG}^{\circledR}$.

\section{Resultados e Discussão}

Ainteração entre o nível de torra e a granulometria afetaram significativamente $(\mathrm{p}<0,05)$ os resultados de teor de água nas duas espécies avaliadas, ao passo que a influência da granulometria não afetou significativamente esta propriedade.

A torra moderadamente escura apresentou menores valores de teor de água quando comparada com a torra média clara. Este fato é esperado, uma vez que um maior tempo no processo de torrefação para se obter um nível de torra mais acentuado acarreta em maior perda de umidade do produto, confirmada pela perda de massa $(4,53 \%$ e 5,36
$\%$ de perda de massa média, respectivamente para nível de torrefação MC e ME). Trabalhos anteriores reportaram esta tendência (SCHMIDT; MIGLIORANZA; PRUDÊNCIO, 2008; BICHO et al., 2012). Os valores de teor de água variaram entre 1,47 e 2,67 (b.s.). Nesta faixa de teor de água, entre 1,00 e 3,70 (b.s.), a agregação da água à estrutura do café torrado é ao nível de monocamada, ou seja, as moléculas de água estão fortemente adsorvidas em locais específicos do produto e a atividade de água é caracterizada como baixa (ILLY; VIANI, 1995), conforme será observado adiante.

A interação entre nível de torrefação e granulometria incorre em diferenças significativas entre as amostras avaliadas $(\mathrm{p}<0,05)$. Tal como observado no parâmetro teor de água, as amostras de café média clara apresentaram maiores valores de atividade de água comparativamente com as amostras moderadamente escura. Estes resultados podem ser explicados devido a torrefação incorrer em reações de pirólise, resultando na produção de $\mathrm{CO}_{2}$, água e substâncias voláteis (PIMENTA et al., 2009), que são facilmente liberadas após a moagem, resultando na baixa atividade de água para o café moído e torrado ME. Os valores de atividade de água variaram entre 0,1493 e 0,3354 .

A Tabela 2 reporta os valores observados do ângulo de repouso dos grãos de $C$. canephora e $C$. arabica para diferentes condições. Observa-se que a interação entre os fatores granulometria e torra $(p<0,05)$ permitiu diferenças significativas entre as amostras avaliadas. Os valores de ângulo de repouso dos grãos de café, em geral, decrescem com o aumento do tamanho das partículas. Este fato é esperado, uma vez que menores partículas permitem o incremento do número total de partículas na massa do produto, aumentando as forças de coesão entre produto/produto (GELDART; ABDULLAH; VERLINDEN, 2009), fornecendo assim maior estabilidade para a massa de grãos, culminando na formação de maiores taludes do produto. 
Tabela 2. Valores médios observados do ângulo de repouso (em graus) dos grãos de Coffea arabica e Coffea canephora e seus respectivos valores de incerteza, em dois níveis de torra ( $\mathrm{MC}$ - média clara; $\mathrm{ME}$ - moderadamente escura) e em quatro granulometrias diferentes ( 0 - inteira, 1 - fina, 2 - média e 3 - grossa).

\begin{tabular}{cccc}
\hline & Amostra & \multicolumn{2}{c}{ Torra } \\
\hline Café & Granulometria & MC & ME \\
\hline \multirow{3}{*}{ Coffea arabica } & 0 & $28,9 \mathrm{D} \mathrm{b} \pm 0,5$ & $34,2 \mathrm{C} \mathrm{a} \pm 0,7$ \\
& 1 & $45,4 \mathrm{~A} \mathrm{a} \pm 0,2$ & $43,0 \mathrm{~A} \mathrm{~b} \pm 0,2$ \\
Coffea & 2 & $39,1 \mathrm{~B} \mathrm{a} \pm 0,3$ & $37,2 \mathrm{~B} \mathrm{~b} \pm 0,2$ \\
canephora & 3 & $36,7 \mathrm{C} \mathrm{a} \pm 0,3$ & $36,6 \mathrm{~B} \mathrm{a} \pm 0,3$ \\
& 0 & $31,0 \mathrm{C} \mathrm{a} \pm 0,4$ & $32,1 \mathrm{C} \mathrm{a} \pm 0,6$ \\
& 1 & $45,0 \mathrm{~A} \mathrm{a} \pm 0,2$ & $44,2 \mathrm{~A} \mathrm{a} \pm 0,2$ \\
& 2 & $35,2 \mathrm{~B} \mathrm{~b} \pm 0,3$ & $38,7 \mathrm{~B} \mathrm{a} \pm 0,3$ \\
\hline
\end{tabular}

Médias seguidas por uma mesma letra maiúscula na coluna e minúscula na linha, para cada espécie de café analisada, não diferem estatisticamente entre si, pelo teste de Tukey a $5 \%$ de probabilidade.

Fonte: Elaboração dos autores.

Em trabalho apresentado por Jong, Hoffmann e Finkers (1999), foi concluído que a escoabilidade de um produto qualquer é inversamente proporcional ao ângulo de repouso; em outras palavras, quanto maior for o ângulo de repouso de uma partícula, maior será a dificuldade do produto em escoar. Esta tendência corrobora com os resultados deste trabalho, em que há um decréscimo dos valores de ângulo de repouso com o aumento da granulometria de café, independentemente do nível de torra ou espécie de café.

A escoabilidade de produtos agrícolas foi também classificada segundo o ângulo de repouso ao passar dos anos (KONSTANCE; ONWULATA; HOLSINGER, 1995; SHITTU; LAWAL, 2007). A última classificação denota que sólidos particulados com até $35^{\circ}$ de ângulo de repouso possuem boa fluidez, os de 35 a $45^{\circ}$ possuem coesividade fraca, os de 45 a $55^{\circ}$ apresentam coesividade e os acima de $55^{\circ}$ são muito coesivos. Portanto, o café pode ser dividido em categorias: grãos de café torrados e inteiros (boa fluidez) e café torrado e moído (coesividade fraca), com os cafés torrados e moído à granulometria fina tendendo a se alterar para a categoria de coesividade ( $\alpha$ entre 45 e $55^{\circ}$ ). Pelos dados apresentados na Tabela 2, nota-se que a granulometria "2" ou média é equivalente à granulometria “" 3 " ou grossa, em termos de ângulo de repouso, na maioria dos casos, independentemente no nível de torra e espécie de café, corroborando com a classificação anterior.

Em grande parte dos produtos agrícolas, espera-se um aumento do ângulo de repouso com o incremento do teor de água do produto devido à aglomeração (no caso de materiais moídos) e ao aumento da área de contato produto/produto (no caso de produtos inteiros). Este comportamento foi observado neste trabalho, em que as amostras MC (maior teor de água), de modo geral, apresentaram valores maiores comparativamente às amostras ME (Tabela 2). Pesquisas com frutos de café (CHANDRASEKAR; VISWANATHAN, 1999) e café em pergaminho (SILVA et al., 2006) concluíram a mesma tendência.

Observa-se ainda que os valores de ângulo de repouso de grãos de café inteiros e com torra branda (MC) foram menores que os valores para os grãos de café inteiros com torra ME. Este fato pode ser explicado pelo aumento da friabilidade das partículas das amostras devido ao maior grau de torrefação, ou seja, são grãos mais susceptíveis de se esfarelarem (MEDEIROS; LANNES, 2010), formando assim partículas menores. 
Os valores de massa específica unitária e aparente, bem como os valores de porosidade, estão inseridos na Tabela 3. A massa específica unitária ou real representa a relação entre a massa de sólido e o volume ocupado pelo produto, excluindo-se os poros abertos e os fechados, assim como os demais espaços vazios (BRITO et al., 2012). Os fatores nível de torrefação e granulometria afetaram significativamente a massa específica unitária.

Tabela 3. Valores médios observados de massa específica unitária $\left(\rho_{\mathrm{u}}\right)$, aparente $\left(\rho_{\text {ap }}\right)$ e porosidade $(\varepsilon)$ dos grãos de Coffea arabica e Coffea canephora e seus respectivos valores de incerteza, em dois níveis de torra (MC - média clara; ME - moderadamente escura) e em quatro granulometrias diferentes ( 0 - inteira, 1 - fina, 2 - média e 3 - grossa).

\begin{tabular}{|c|c|c|c|c|c|c|c|}
\hline \multicolumn{2}{|c|}{ Amostra } & \multicolumn{2}{|c|}{$\rho_{\mathrm{u}}\left(\mathrm{kg} \mathrm{m}^{-3}\right)$} & \multicolumn{2}{|c|}{$\rho_{\text {ap }}\left(\mathrm{kg} \mathrm{m}^{-3}\right)$} & \multicolumn{2}{|c|}{$\varepsilon(\%)$} \\
\hline Café & Granulometria & $\mathrm{MC}$ & $\mathrm{ME}$ & $\mathrm{MC}$ & $\mathrm{ME}$ & $\mathrm{MC}$ & $\mathrm{ME}$ \\
\hline \multirow{4}{*}{$\begin{array}{l}\text { Coffea } \\
\text { arabica }\end{array}$} & 0 & $\begin{array}{c}705,17 \mathrm{D} \mathrm{a} \\
\pm 0,04\end{array}$ & $\begin{array}{c}680,69 \mathrm{D} \mathrm{b} \\
\pm 0,04\end{array}$ & $\begin{array}{c}359,04 \mathrm{~A} \mathrm{a} \\
\pm 3,57\end{array}$ & $\begin{array}{c}342,26 \mathrm{~A} \mathrm{~b} \\
\pm 1,94\end{array}$ & $\begin{array}{c}49,1 \mathrm{D} \mathrm{b} \\
\pm 0,10\end{array}$ & $\begin{array}{c}49,7 \mathrm{D} \mathrm{a} \\
\pm 0,18\end{array}$ \\
\hline & 1 & $\begin{array}{c}1096,47 \mathrm{~A} \mathrm{~b} \\
\quad \pm 0,03\end{array}$ & $\begin{array}{c}1178,04 \mathrm{~A} \mathrm{a} \\
\quad \pm 0,03\end{array}$ & $\begin{array}{c}315,04 \text { B C } \\
b \pm 5,79\end{array}$ & $\begin{array}{c}321,38 \mathrm{~B} \mathrm{a} \\
\pm 7,20\end{array}$ & $\begin{array}{c}71,3 \mathrm{~A} \mathrm{~b} \\
\pm 0,37\end{array}$ & $\begin{array}{c}72,7 \mathrm{~A} \mathrm{a} \\
\pm 0,06\end{array}$ \\
\hline & 2 & $\begin{array}{c}952,95 \mathrm{~B} \mathrm{a} \\
\pm 0,02\end{array}$ & $\begin{array}{l}939,59 \mathrm{~B} \mathrm{~b} \\
\quad \pm 0,02\end{array}$ & $\begin{array}{c}312,92 \mathrm{~B} \mathrm{a} \\
\pm 1,11\end{array}$ & $\begin{array}{c}316,34 \mathrm{~B} \mathrm{a} \\
\pm 1,66\end{array}$ & $\begin{array}{c}67,2 \mathrm{~B} \mathrm{a} \\
\pm 0,08\end{array}$ & $\begin{array}{c}66,3 \mathrm{~B} \mathrm{~b} \\
\pm 0,16\end{array}$ \\
\hline & 3 & $\begin{array}{c}868,64 \mathrm{C} \text { a } \\
\pm 0,02\end{array}$ & $\begin{array}{c}831,59 \mathrm{C} \mathrm{b} \\
\quad \pm 0,02 \\
\end{array}$ & $\begin{array}{c}319,91 \mathrm{C} \text { a } \\
\pm 1,37\end{array}$ & $\begin{array}{c}308,68 \mathrm{Cb} \\
\pm 2,08\end{array}$ & $\begin{array}{c}63,2 \mathrm{C} \mathrm{a} \\
\pm 0,18 \\
\end{array}$ & $\begin{array}{c}62,9 \mathrm{C} \text { a } \\
\pm 0,21 \\
\end{array}$ \\
\hline \multirow{4}{*}{$\begin{array}{c}\text { Coffea } \\
\text { canephora }\end{array}$} & 0 & $\begin{array}{l}872,47 \mathrm{D} \text { a } \\
\quad \pm 0,03\end{array}$ & $\begin{array}{c}761,75 \mathrm{D} \mathrm{b} \\
\pm 0,03\end{array}$ & $\begin{array}{c}406,88 \mathrm{~A} \mathrm{a} \\
\quad \pm 2,91\end{array}$ & $\begin{array}{c}349,66 \mathrm{~A} \mathrm{~b} \\
\pm 1,33\end{array}$ & $\begin{array}{c}53,4 \mathrm{D} \mathrm{b} \\
\pm 0,03\end{array}$ & $\begin{array}{c}54,1 \mathrm{D} \mathrm{a} \\
\pm 0,18\end{array}$ \\
\hline & 1 & $\begin{array}{c}1221,53 \mathrm{~A} \mathrm{a} \\
\quad \pm 0,09\end{array}$ & $\begin{array}{c}1178,45 \mathrm{~A} \mathrm{~b} \\
\quad \pm 0,05\end{array}$ & $\begin{array}{c}362,05 \mathrm{~B} \mathrm{a} \\
\quad \pm 2,92\end{array}$ & $\begin{array}{c}314,02 \mathrm{~B} \mathrm{~b} \\
\pm 4,95\end{array}$ & $\begin{array}{c}70,4 \mathrm{~A} \mathrm{~b} \\
\pm 0,14\end{array}$ & $\begin{array}{c}73,4 \mathrm{~A} \mathrm{a} \\
\pm 0,43\end{array}$ \\
\hline & 2 & $\begin{array}{c}1017,85 \mathrm{~B} \mathrm{a} \\
\quad \pm 0,09\end{array}$ & $\begin{array}{c}910,62 \mathrm{~B} \mathrm{~b} \\
\quad \pm 0,02\end{array}$ & $\begin{array}{c}361,54 \mathrm{~B} \mathrm{a} \\
\quad \pm 1,71\end{array}$ & $\begin{array}{c}324,15 \mathrm{C} \mathrm{b} \\
\quad \pm 2,93\end{array}$ & $\begin{array}{c}64,5 \mathrm{~B} \mathrm{a} \\
\pm 0,13\end{array}$ & $\begin{array}{c}64,4 \mathrm{~B} \mathrm{a} \\
\pm 0,21\end{array}$ \\
\hline & 3 & $\begin{array}{c}947,95 \mathrm{C} \mathrm{a} \\
\quad \pm 0,04\end{array}$ & $\begin{array}{c}805,96 \mathrm{C} \mathrm{b} \\
\quad \pm 0,05\end{array}$ & $\begin{array}{c}359,31 \mathrm{~B} \mathrm{a} \\
\pm 0,74\end{array}$ & $\begin{array}{c}311,23 \mathrm{~B} \mathrm{~b} \\
\pm 1,26\end{array}$ & $\begin{array}{c}62,1 \mathrm{C} \mathrm{a} \\
\pm 0,03\end{array}$ & $\begin{array}{c}61,4 \mathrm{C} \mathrm{b} \\
\pm 0,09\end{array}$ \\
\hline
\end{tabular}

Médias seguidas por uma mesma letra maiúscula na coluna e minúscula na linha, para cada espécie de café e propriedade analisada, não diferem estatisticamente entre si, pelo teste de Tukey a $5 \%$ de probabilidade.

Fonte: Elaboração dos autores.

De acordo com a Tabela 3, a massa específica unitária máxima e a mínima foi de 1221,53 e 680,69 $\mathrm{kg} \mathrm{m}^{-3}$, associadas às torrefações média clara e moderadamente escura, respectivamente. Em geral, os valores de $\rho_{\mathrm{u}}$ foram maiores para o nível de torra média clara, independentemente da espécie de café. Este fato é explicado pela variação de volume e de massa durante a torrefação: nível de torra mais brando leva a um menor aumento de volume do produto e perda de massa, obtendo-se assim maiores valores de massa específica unitária. Singh et al. (1997), em seu trabalho com café mexicano e colombiano, também concluíram que níveis de torrefação maiores acarretam em decréscimo nos valores de massa específica unitária. Vargas-Elías (2011) reportou a diminuição da massa específica unitária em café com nível de torra mais acentuado devido à maior perda de massa do produto.

Outro fato relativo ao nível de torrefação se baseia ao nível celular. Segundo Licciardi et al. (2005), os triglicerídeos (óleos) do grão do café são pouco afetados pela torrefação, sofrendo apenas pequena hidrólise e decomposição, com liberação de ácidos graxos e formação de produtos voláteis. No entanto, nas torrações mais escuras, muitas células sofrem rompimento e o óleo pode migrar para a superfície do produto (FRANÇA et al., 2001), ocorrendo assim, maior perda de massa, resultando 
na diminuição dos valores de massa específica. $\mathrm{O}$ teor de óleo que afeta a massa específica também foi verificado em trabalho com leite em pó (FITZPATRICK et al., 2004).

Observa-se na Tabela 3 que os valores de massa específica unitária decresceram com o aumento do tamanho das partículas (granulometria). Em um dado volume constante, os cafés com moagem mais fina se aglomeram mais intensamente, resultando numa massa maior para aquele volume, ao passo que partículas maiores há uma menor aglomeração, culminando em menor massa e, consequentemente, menores valores de massa específica unitária. SINGH et al. (1997) relataram comportamento similar ao longo do processo de torrefação de café.

A massa específica aparente é a razão entre a massa do sólido e o volume do sólido contendo poros. Os valores de massa específica aparente foram influenciados pela interação entre o nível de torrefação e a granulometria $(\mathrm{p}<0,05)$, variando entre 311,23 e 406,88 $\mathrm{kg} \mathrm{m}^{-3}$. Estes valores estão na faixa de 300 a $450 \mathrm{~kg} \mathrm{~m}^{-3}$ reportada por Illy e Viani (1995) para café torrado.

Essa propriedade, tal como ocorreu com a massa específica unitária, foi maior para o produto torrado ao nível média clara. Este fato está associado ao incremento de volume do produto e perda de massa devido ao maior tempo de torrefação. Borges et al. (2004) explicam que a alteração no volume está relacionado com o aumento da pressão interna das estruturas das células que ocorre durante a transferência de calor e nas reações do processo de pirólise, no qual são mais intensas em torrefação prolongada (ME). Ademais, a massa do produto também influencia na diminuição dessa propriedade, em que maior tempo de torrefação acaba por ocorrer maior perda de massa. Diferentes trabalhos com café relataram esta tendência (ILLY; VIANI, 1995; SINGH et al., 1997; PITTIA; DALLA ROSA; LERICI, 2001; MWITHIGA; JINDAL, 2003; MENDONÇA; FRANCA; OLIVEIRA, 2009; VARGAS-ELÍAS, 2011).
Nas diferentes granulometrias estudadas, nota-se que os grãos de café inteiros obtiveram maiores valores de massa específica aparente. Esta ocorrência está diretamente relacionada ao conteúdo de componentes voláteis que por sua vez está relacionada à massa total do produto. Grãos inteiros liberam $\mathrm{CO}_{2}$ lentamente, sendo necessários cerca de 30 dias para sua total liberação, resultando em maiores valores de massa, ao passo que $70 \%$ de $\mathrm{CO}_{2}$ são imediatamente liberados após a moagem (ILLY; VIANI, 1995).

Para os cafés torrados e moídos, percebe-se, em termos gerais, que a massa específica aparente decresce com o aumento do tamanho das partículas. Yan e Barbosa-Canovas (1997), estudando a compressibilidade de alimentos pulverulentos, relataram esta mesma relação entre granulometria e massa específica aparente. Estes autores explicam que o aumento do tamanho das partículas leva à diminuição do volume ocupado pelo produto; em outras palavras, há maior quantidade de poros, ou seja, menor massa de produto em dado volume constante, resultando em menores valores de massa específica aparente.

A interação entre o nível de torrefação e a granulometria do café foi significativa $(\mathrm{p}<0,05)$ para os resultados de porosidade. Entre os níveis de torra, percebe-se que, de um modo geral, a torra $\mathrm{ME}$ apresenta porosidade inferior a torra MC (Tabela 2). Este fato está intimamente ligado à friabilidade do café torrado $\mathrm{ME}$, pois quanto maior é o nível de torrefação, maior é a friabilidade do produto (MEDEIROS; LANNES, 2010), resultando em partículas menores após a moagem, levando à menor quantidade de vazios na massa pulverulenta.

A porosidade variou de modo evidente entre os diferentes níveis de granulometria, sendo o café torrado e moído fino o que obteve os maiores valores de porosidade, decrescendo ao passo que a granulometria aumenta até atingir os menores valores para os grãos de café inteiro e torrado. Esta tendência não era esperada, uma vez que pesquisas 
anteriores relataram um comportamento diretamente proporcional entre granulometria e porosidade (GRELLIER; RIVIERE; RENAULT, 1999; ANJOS et al., 2001). No entanto, o comportamento observado pode ser em razão da aglomeração do produto. Schubert (1987) relata que a porosidade aumenta com o decréscimo do tamanho das partículas devido à adesão entre partículas permitir uma estrutura desprendida da massa. Pegg e Shahidi (2007) complementam este trabalho explicando que produtos com menor granulometria tendem a se aglomerar, formando agregados maiores que o próprio grão, resultando em porosidade elevada, conforme constatado neste trabalho.
Para os grãos de café torrado e inteiro, os valores de porosidade variaram entre 49,1 e $54,1 \%$ encontram-se na faixa de valores observada para a maioria dos grãos, sendo também compatível com prévios estudos com café (CHANDRASEKAR; VISWANATHAM, 1999; VARGAS-ELÍAS, 2011).

Por meio da análise de variância constatouse que a interação entre a granulometria e o nível de torrefação foi significativa $(\mathrm{p}<0,05)$ para os resultados de cor. Tendo em vista a torrefação realizada e os resultados obtidos, o índice ângulo hue e a coordenada $L^{*}$ serão abordados a seguir. A Tabela 4 relata os valores destes de acordo com o nível de torrefação, espécie de café e granulometria.

Tabela 4. Valores médios da coordenada colorimétrica L* e índice colorimétrico ângulo hue (h*) dos grãos de Coffea arabica e Coffea canephora e seus respectivos valores de incerteza, em dois níveis de torra (MC - média clara; ME moderadamente escura) e em quatro granulometrias diferentes ( 0 - inteira, 1 - fina, 2 - média e 3 - grossa).

\begin{tabular}{cccccc}
\hline \multicolumn{2}{c}{ Amostra } & \multicolumn{2}{c}{$\mathrm{L}^{*}$} & \multicolumn{2}{c}{$\mathrm{h}^{*}$} \\
\hline Café & Granulometria & \multicolumn{1}{c}{$\mathrm{MC}$} & $\mathrm{ME}$ & $\mathrm{MC}$ & $\mathrm{ME}$ \\
Coffea & 0 & $13,6 \mathrm{C} \mathrm{a} \pm 0,2$ & $12,8 \mathrm{C} \mathrm{b} \pm 0,9$ & $28,9 \mathrm{C} \mathrm{a} \pm 1,1$ & $24,0 \mathrm{C} \mathrm{b} \pm 2,8$ \\
arabica & 1 & $17,4 \mathrm{~A} \mathrm{a} \pm 0,2$ & $16,9 \mathrm{~B} \mathrm{a} \pm 0,1$ & $33,4 \mathrm{~A} \mathrm{a} \pm 0,2$ & $34,6 \mathrm{~A} \mathrm{a} \pm 3,0$ \\
& 2 & $17,2 \mathrm{AB} \mathrm{a} \pm 0,1$ & $17,6 \mathrm{AB} \mathrm{a} \pm 0,2$ & $35,8 \mathrm{~A} \mathrm{a} \pm 0,4$ & $37,8 \mathrm{AB} \mathrm{a} \pm 0,9$ \\
& 3 & $17,5 \mathrm{AB} \mathrm{b} \pm 0,1$ & $18,5 \mathrm{~A} \mathrm{a} \pm 0,5$ & $36,9 \mathrm{~A} \mathrm{~b} \pm 0,4$ & $40,8 \mathrm{~B} \mathrm{a} \pm 1,4$ \\
\hline \multirow{2}{*}{ Coffea } & 0 & $18,3 \mathrm{C} \mathrm{a} \pm 0,9$ & $13,2 \mathrm{~B} \mathrm{~b} \pm 0,8$ & $40,2 \mathrm{~B} \mathrm{a} \pm 1,1$ & $24,8 \mathrm{C} \mathrm{b} \pm 2,4$ \\
canephora & 1 & $22,3 \mathrm{~A} \mathrm{a} \pm 0,4$ & $18,4 \mathrm{~A} \mathrm{~b} \pm 0,3$ & $45,0 \mathrm{~A} \mathrm{a} \pm 0,5$ & $36,0 \mathrm{~B} \mathrm{~b} \pm 1,1$ \\
& 2 & $20,4 \mathrm{~B} \mathrm{a} \pm 0,2$ & $18,8 \mathrm{~A} \mathrm{~b} \pm 0,6$ & $42,8 \mathrm{AB} \mathrm{a} \pm 0,2$ & $41,7 \mathrm{~A} \mathrm{a} \pm 1,6$ \\
\hline
\end{tabular}

Médias seguidas por uma mesma letra maiúscula na coluna e minúscula na linha, para cada espécie de café e coordenada colorimétrica, não diferem estatisticamente entre si, pelo teste de Tukey a $5 \%$ de probabilidade.

Fonte: Elaboração dos autores.

Analisando a Tabela 4, conclui-se que em todas as amostras avaliadas houve diferenciação significativa entre o grão de café torrado inteiro e as amostras de café torrado e moído, independentemente da espécie, nível de torrefação e coordenada colorimétrica. Esta ocorrência se deve à maior torrefação da parte exterior dos grãos em vista ao interior dos grãos (ILLY; VIANI, 1995) e, após a moagem, a mistura das partículas mais torradas com as menos torradas resulta na diferenciação anteriormente citada. Borges et al. (2002) concluíram este mesmo fato de modo a explicar as magnitudes inferiores das coordenadas colorimétricas dos grãos de café torrados sem serem submetidos à moagem em vista às coordenadas dos cafés moídos. Os valores da coordenada $L^{*}$ variaram entre 12,77 e 22,35, corroborando com trabalho apresentado por Mwithiga e Jingal (2003), no qual os autores encontraram valores entre 13 e 21 para essa coordenada. Borges et al. (2002) explicaram a tendência de decréscimo da luminosidade de acordo com a intensidade de torra em função do escurecimento dos grãos devido à caramelização dos açúcares e reações de Maillard. 
Para o ângulo hue, houve comportamentos distintos devido à espécie de café utilizada, entre os dois níveis de torrefação. C. arabica apresentaram diferenças significativas apenas para o café inteiro e o moído grosso, ao passo que para C. canephora essas diferenças ocorreram em 87,5 \% das combinações granulometria e nível de torrefação. Bicho et al. (2012) relataram comportamento desigual entre as duas espécies de café, tal como neste trabalho.

Para visualizar as diferenças colorimétricas entre as amostras, a Figura 2 foi elaborada de modo a permitir a representação dos pontos obtidos da plotagem dos valores de ângulo hue em função dos valores de croma no espaço Hunter, localizando assim a exata posição das cores das diferentes amostras aqui estudadas. Observa-se na Figura 2 que a maior diferença visual é do Coffea canephora na torra clara, seguido desta mesma espécie na torra escura. Já as amostras de Coffea arabica se concentraram em uma parte do espaço cromático de Hunter, indicando a pouca diferenciação entre as torras e as granulometrias para esta espécie.

Figura 2. Localização das cores dos grãos de café torrados, inteiros e moídos, no espaço cromático de Hunter, utilizando as coordenadas croma e ângulo hue.
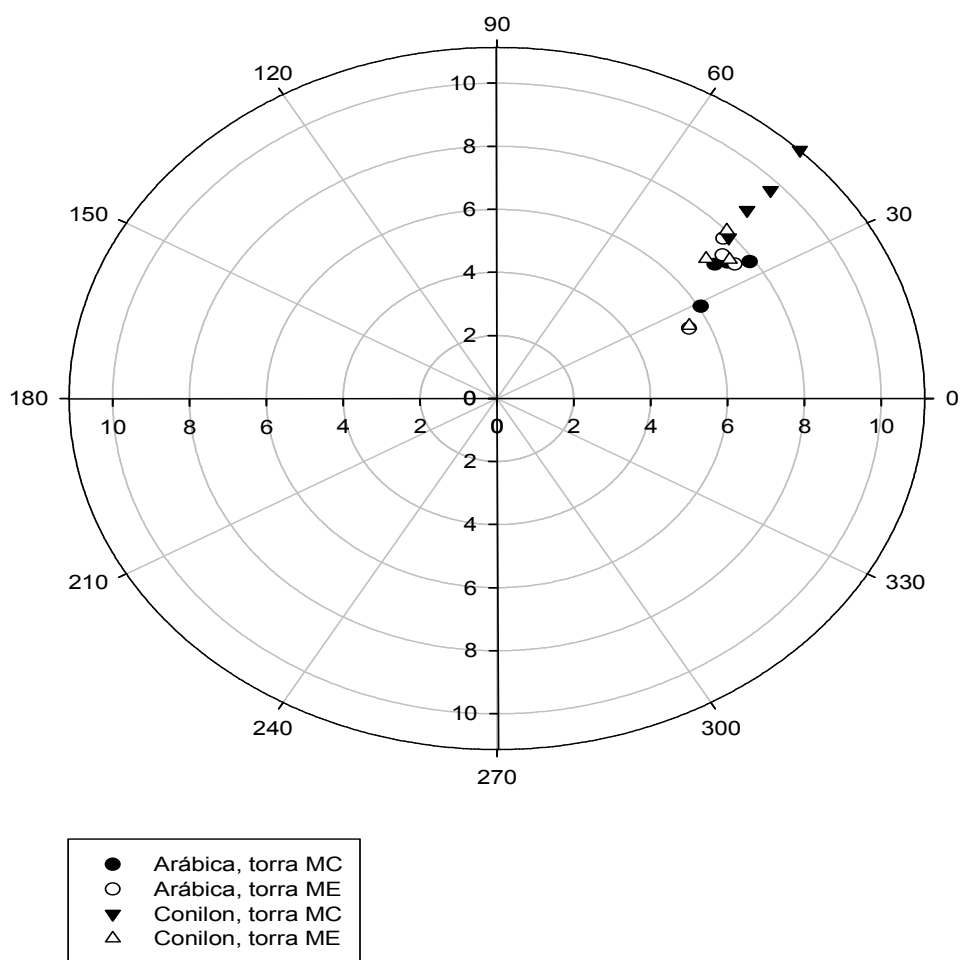

Fonte: Elaboração dos autores.

Os diâmetros $\mathrm{D}_{10}, \mathrm{D}_{50}$ e $\mathrm{D}_{90}$, que equivalem, respectivamente, a 10, 50 e $90 \%$ do total da massa do material analisado com diâmetro abaixo do valor encontrado estão apresentados na Tabela 5. Os resultados indicam que os cafés torrados e moídos nas granulometrias média e grossa são próximos em termos granulométricos, uma vez que a diferença entre estas amostras, independentemente da espécie de café, é baixa. Entretanto, o café torrado e moído na granulometria fina difere das amostras restantes, em todas as três faixas de diâmetros. 
Tabela 5. Diâmetros das amostras dos grãos de Coffea arabica e Coffea canephora e seus respectivos valores de incerteza, em dois níveis de torra ( $\mathrm{MC}$ - média clara; $\mathrm{ME} \mathrm{-} \mathrm{moderadamente} \mathrm{escura)} \mathrm{e} \mathrm{em} \mathrm{três} \mathrm{granulometrias} \mathrm{diferentes}$ ( 1 - fina, 2 - média e 3 - grossa).

\begin{tabular}{cccccccc}
\hline & Amostra & \multicolumn{2}{c}{$\mathrm{D}_{10}(\mu \mathrm{m})$} & \multicolumn{2}{c}{$\mathrm{D}_{50}(\mu \mathrm{m})$} & \multicolumn{2}{c}{$\mathrm{D}_{90}(\mu \mathrm{m})$} \\
\hline Café & Granulometria & $\mathrm{MC}$ & $\mathrm{ME}$ & $\mathrm{MC}$ & $\mathrm{ME}$ & $\mathrm{MC}$ & $\mathrm{ME}$ \\
\hline \multirow{2}{*}{ Coffea } & 1 & 5,96 & 7,22 & 185,05 & 276,72 & 445,74 & 452,95 \\
arabica & 2 & 3,87 & 3,39 & 42,80 & 38,18 & 349,43 & 339,50 \\
& 3 & 3,61 & 3,46 & 41,00 & 46,19 & 349,34 & 367,40 \\
\hline \multirow{2}{*}{ Coffea } & 1 & 10,64 & 12,23 & 157,28 & 287,05 & 442,32 & 454,49 \\
canephora & 2 & 7,58 & 7,47 & 60,93 & 55,01 & 390,52 & 373,92 \\
& 3 & 7,07 & 6,32 & 64,35 & 72,23 & 384,25 & 402,05 \\
\hline
\end{tabular}

Fonte: Elaboração dos autores.

Os resultados na Tabela 5 mostram que as amostras de café nas granulometrias 2 e 3 são materiais mais finos que as amostras de café moídas na granulometria 1 ou fina, fato que difere do observado durante as análises. Este comportamento é explicado pela metodologia das análises, em que o pó de café era diluído com água destilada através de uma malha de abertura $150 \mu \mathrm{m}$. Nas amostras com granulometrias 2 e 3 eram necessários maiores volumes de amostra, uma vez que grande parte do pó ficava retido, resultando na passagem apenas do material fino presente, mascarando assim os resultados da distribuição granulométrica. Dessa forma, a granulometria a laser não é indicada para a análise granulométrica de café torrado e moído.

Os valores de ângulo de atrito interno e efetivo ângulo de atrito interno estão apresentados na Tabela 6. O ângulo de atrito interno $\left(\mathrm{f}_{\mathrm{i}}\right)$ é a força tangencial necessária para vencer o atrito entre dois corpos (GAGGERO; TREIN; IPPOLITI, 2002). Segundo estes mesmos autores, ele é proporcional à força normal sobre os grãos, sendo a força contrária constituída por uma mistura de pressões de escorregamento e de rolamento entre grãos. Nascimento (2008) relata que este ângulo está intimamente ligado às condições internas do produto armazenado, dependendo do nível médio de pressões aplicado a todos os grãos.

Os valores de $f_{i}$ variaram entre 20,3 e $41,2^{\circ}$ para o Coffea arabica e entre 21,5 e $34,2^{\circ}$ para o Coffea canephora. Especificamente para a primeira espécie, houve diferença entre as amostras com torra $\mathrm{MC}$ e a ME, nas amostras de café inteiro e com granulometria moído grossa, sendo a torra MC a que possibilitou maiores valores deste parâmetro. Esta tendência está intimamente ligada ao teor de água, em que produtos com maiores valores desta propriedade apresentam uma maior força de coesão entre as partículas (aumentando o atrito interno), podendo também ocorrer um aumento da rugosidade da superfície do produto, incremento a resistência ao deslizamento entre partículas (SILVA et al., 2006), resultando nos maiores valores de $f_{i}$. Comportamento similar foi encontrado em trabalho com avelãs (KIBAR; ÖZTÜRK, 2009). 
Tabela 6. Valores médios de ângulo de atrito interno (Фi) e efetivo ângulo de atrito interno (Фe) dos grãos de Coffea arabica e Coffea canephora e seus respectivos valores de incerteza, em dois níveis de torra (MC - média clara; ME moderadamente escura) e em quatro granulometrias diferentes ( 0 - inteira, 1 - fina, 2 - média e 3 - grossa).

\begin{tabular}{cccccc}
\hline & Amostra & \multicolumn{2}{c}{$\Phi \mathrm{i}\left({ }^{\circ}\right)$} & \multicolumn{2}{c}{$\Phi \mathrm{e}\left(^{\circ}\right)$} \\
\hline Café & Granulometria & $\mathrm{MC}$ & $\mathrm{ME}$ & $\mathrm{MC}$ & $\mathrm{ME}$ \\
\hline \multirow{3}{*}{ Coffea } & 0 & $41,2 \mathrm{~A} \mathrm{a}$ & $30,5 \mathrm{~A} \mathrm{~b}$ & $41,2 \mathrm{~A} \mathrm{a}$ & $31,0 \mathrm{~A} \mathrm{~b}$ \\
arabica & 1 & $28,3 \mathrm{~B} \mathrm{a}$ & $26,0 \mathrm{~A} \mathrm{a}$ & $34,9 \mathrm{~B} \mathrm{a}$ & $33,1 \mathrm{~A} \mathrm{a}$ \\
& 2 & $27,9 \mathrm{~B} \mathrm{a}$ & $27,0 \mathrm{~A} \mathrm{a}$ & $32,2 \mathrm{~B} \mathrm{a}$ & $32,0 \mathrm{~A} \mathrm{a}$ \\
& 3 & $30,8 \mathrm{~B} \mathrm{a}$ & $20,3 \mathrm{AB} \mathrm{b}$ & $32,7 \mathrm{~B} \mathrm{a}$ & $31,3 \mathrm{~A} \mathrm{a}$ \\
\hline \multirow{2}{*}{ Coffea } & 0 & $34,2 \mathrm{~A} \mathrm{a}$ & $29,8 \mathrm{~A} \mathrm{a}$ & $34,2 \mathrm{~A} \mathrm{a}$ & $30,0 \mathrm{AB} \mathrm{b}$ \\
canephora & 1 & $28,1 \mathrm{~A} \mathrm{a}$ & $28,1 \mathrm{~A} \mathrm{a}$ & $33,6 \mathrm{~A} \mathrm{a}$ & $33,2 \mathrm{~A} \mathrm{a}$ \\
& 2 & $25,1 \mathrm{~A} \mathrm{a}$ & $22,7 \mathrm{~A} \mathrm{a}$ & $31,2 \mathrm{~A} \mathrm{a}$ & $28,3 \mathrm{~B} \mathrm{a}$ \\
& 3 & $26,1 \mathrm{~A} \mathrm{a}$ & $21,5 \mathrm{~A} \mathrm{a}$ & $32,6 \mathrm{~A} \mathrm{a}$ & $27,8 \mathrm{~B} \mathrm{~b}$ \\
\hline
\end{tabular}

Médias seguidas por uma mesma letra maiúscula na coluna e minúscula na linha, para cada espécie de café e cada ângulo, não diferem estatisticamente entre si, pelo teste de Tukey a $5 \%$ de probabilidade.

Fonte: Elaboração dos autores.

O comportamento de $f_{i}$ foi de diminuição com o aumento do tamanho das partículas (granulometria), para os casos das amostras de café torrado e moído. Uma possível explicação para este comportamento tem como fundamento o número global de pontos de contato entre as partículas de café. Uma moagem mais fina acarreta em maior quantidade de partículas, apesar de menores, que acabam por incrementar o número de pontos de contato entre as mesmas, levando a um maior valor de resistência de deslizamento entre as partículas, culminando nos maiores valores de $f_{i}$ encontrados no presente trabalho. Esta tendência também foi encontrada por Podczeck e Miah (1996) trabalhando com oito diferentes pós com distintas granulometrias e formas.

Portanto, as amostras de café torradas MC apresentam, de modo geral, maior susceptibilidade em desenvolver coesão quando armazenados (LOPES NETO; NASCIMENTO; SILVA, 2009), maior resistência ao fluxo (CHEVANAN et al., 2009) e maiores problemas com procedimentos pós-colheita que requeiram movimentação do produto, quando comparadas com as amostras de café torradas ME.
Segundo Naka (2010), para o cálculo da inclinação da tremonha, de modo a se evitar a formação de obstruções que venham a impedir o correto escoamento do produto armazenado, são necessários os valores de $\mathrm{f}_{\mathrm{i}}$ e do efetivo ângulo de atrito interno $\left(\mathrm{f}_{\mathrm{e}}\right)$. Este último é o ângulo formado pela reta que passa pela origem com o eixo das tensões normais.

A Tabela 6 reporta uma faixa de valores de $f_{e}$ entre 31,0 e $41,2^{\circ}$ e entre 27,8 e $34,2^{\circ}$ para as amostras de café das espécies Coffea arabica e Coffea canephora, respectivamente. Tal como $\mathrm{f}_{i}$, os valores de $f_{e}$ foram maiores para a torrefação mais branda (MC) e tendência de decréscimo com o aumento do tamanho das partículas. Este comportamento é explicado pelo teor de água e pelo número global de pontos de contato entre as partículas de café. Elevados teores de água leva o produto a apresentar maior força de coesão entre as partículas, maior rugosidade da superfície do produto, aumentando-se assim a resistência ao deslizamento entre partículas. A moagem mais fina acarreta em maior quantidade de partículas, aumentando o número de pontos de contato entre as mesmas e consequentemente incrementando a resistência de deslizamento entre as partículas. 


\section{Conclusões}

Com base nos resultados obtidos e nas condições em que foi realizado o experimento, conclui-se que:

- A granulometria não afetou significativamente o teor de água de café, independentemente da espécie e nível de torrefação;

- O nível de torrefação apresentou relação inversa significativa com o teor de água de café, em que quanto maior o grau de torra, menores são os valores desta propriedade física;

- O grau de torrefação e as diferentes granulometrias empregadas apresentaram influência sobre a atividade de água de café, em que amostras moídas de forma mais grossa combinada com torra mais branda obtém alta atividade de água;

- Valores de ângulo de repouso, ângulo de atrito interno e efetivo ângulo de atrito interno de café torrado, inteiro e moído, decrescem com o aumento da granulometria;

- A torra média clara fornece maiores valores de ângulo de repouso, ângulo de atrito interno e o efetivo ângulo de atrito interno de café torrado, quando comparada com a torra moderadamente escura;

- A torrefação moderadamente escura fornece menores valores de massa específica unitária e aparente, bem como para a porosidade;

- As propriedades massa específica unitária, aparente e porosidade aumentam com o incremento do grau de moagem de café;

- A cor de café torrado, inteiro e moído, pode ser relacionada com a coordenada colorimétrica $\mathrm{L}^{*}$ e pelo ângulo hue;

- A técnica de granulometria a laser não é adequada para a análise granulométrica de café torrado e moído.

\section{Agradecimentos}

Ao $\mathrm{CNPq}$ pela bolsa e pelo auxílio (Edital Universal CNPq nº 14/2012).

\section{Referências}

ASSOCIAÇÃO BRASILEIRA DA INDÚSTRIA DE CAFÉ-ABIC. Norma de qualidade recomendável e boas práticas de fabricação de cafés torrados em grão e cafés torrados e moídos. 2013. (Revisão, 26). Disponível em: $<$ http://www.abic.com.br/publique/media/Norma $\% 20$ de\%20qualidade.pdf $>$. Acesso em: 15 out. 2013.

ANJOS, V. D. A.; FARINASSI, F. M.; SALLES, M. A.; MENEZES, S. G. Desenvolvimento de um sistema utilizado de café torrado e moído: otimização da operação de prensagem. In: SIMPÓSIO DE PESQUISA DOS CAFÉS DO BRASIL, 2., 2001, Vitória. Resumos... Vitória: [s. n], 2001. p. 1-9.

BICHO, N. C.; LEITÃO, A. E.; RAMALHO, J. C.; LIDON, F. C. Use of colour parameters for roasted coffee assessment. Ciência e Tecnologia de Alimentos, Campinas, v. 32, n. 3, p. 436-442, 2012.

BRITISH MATERIALS HANDLING BOARD BMHB. Draft code of practice for the design of silos, bins, bunkers and hoppers. Inglaterra, 1985. $101 \mathrm{p}$.

BORGES, M. L. A.; FRANÇA, A. S.; OLIVEIRA, L. S.; CORRÊA, P. C.; GLÓRIA, M. B. A. Estudo da variação da coloração de café arábica durante a torra em diferentes condições de aquecimento. Revista Brasileira de Armazenamento, Viçosa, n. 5, p. 3-8, 2002. Especial Café.

BORGES, M. L. A.; MENDONÇA, J. C. F.; FRANCA, A. S.; OLIVEIRA, L. S.; CORRÊA, P. C. Efeito da torração em parâmetros físicos de cafés de diferentes qualidades. Revista Brasileira de Armazenamento, Viçosa, n. 8, p. 6-13, 2004. Especial Café.

BRASIL. Ministério da Agricultura, Pecuária e Abastecimento. Regras para análises de sementes. Ministério da Agricultura, Pecuária e Abastecimento. Secretaria de Defesa Agropecuária. MAPA/ACS, Brasília, 2009. 399 p.

BRITO, J. G.; SILVA, E. F. M.; COSTA, T. L.; ROCHA, A. P. T.; SILVA, O. S. Propriedades físicas do cominho em pó comercializado nas feiras livres da Paraíba. Revista Verde, Mossoró, v. 7, n. 1, p. 206-211, 2012.

CHANDRASEKAR, V.; VISWANATHAN, R. Physical and thermal properties of coffee. Journal of Agricultural Engineering Research, Filadélfia, v. 73, n. 3, p. 227-234, 1999. 
CHEVANAN, N.; WOMAC, A. R.; BITRA, V. S. P.; YODER, D. C.; SOKHANSANJ, S. Flowability parameters for chopped switchgrass, wheat straw and corn stover. Powder Technology, Filadélfia, v. 193, n. 1, p. 79-86, 2009.

FITZPATRICK, J. J.; IQBAL, T.; DELANEY, C.; TWOMEY, T.; KEOGH, M. K. Effect of powder properties and storage conditions on the flowability of milk powders with different fat contents. Journal of Food Engineering, Filadélfia, v. 64, n. 4, p. 435-444, 2004.

FRANÇA, A. S.; OLIVEIRA, L. S.; BORGES, M. L. A.; VITORINO, M. D. Evolução da composição do extrato aquoso de café durante o processo de torrefação. Revista Brasileira de Armazenamento, Viçosa, n. 2, p. 37-47, 2001. Especial Café.

GAGGERO, M. R.; TREIN, C. R.; IPPOLITI, G. Influência de sistemas de preparo e pastejo nas características físicas do solo. Revista do Programa de Ciências Agro-Ambientais, Alta Floresta, v. 1, n. 1, p. 1-16, 2002.

GELDART, D.; ABDULLAH, E. C.; VERLINDEN, A. Characterisation of dry powders. Powder Technology, Filadélfia, v. 190, n. 1-2, p. 70-74, 2009.

GRELliER, P.; RIVIERE, L. M.; RENAULT, P. Transfer and water-retention properties of seed-pelleting materials. European Journal of Agronomy, Filadélfia, v. 10, n. 1, p. 57-65, 1999.

ILLY, A.; VIANI, R. Espresso coffee: the chemistry of quality. London: Academic Press, 1995. 253 p.

JENIKE, A. W.; JOHANSON, J. Flow factor Tester and consolidating bench operating instructions. North Billerica, Massachusetts: Jenike \& Johonson Inc, 1979. $40 \mathrm{p}$.

JONG, J. A. H.; HOFFMANN, A. C.; FINKERS, H. J. Properly determine powder flowability to maximize plant output. Chemical Engineering Progress, Nova Iorque, v. 95, n. 4, p. 25-34, 1999.

KIBAR, H.; ÖZTÜRK, T. The effect of moisture content on the physico-mechanical properties of some hazelnut varieties. Journal of Stored Products Research, Filadélfia, v. 45, n. 1, p. 14-18, 2009.

KONSTANCE, R. P.; ONWULATA, C. I.; HOLSINGER, V. H. Flow properties of spray-dried encapsulated butteroil. Journal of Food Science, Maiden, v. 60, n. 4, p. 841-844, 1995.

LICCIARDI, R.; PEREIRA, R. G. F. A.; MENDONÇA, L. M. V. L.; FURTADO, E. F. Avaliação físico-química de cafés torrados e moídos, de diferentes marcas comerciais, da região sul de minas gerais. Ciência e Tecnologia de Alimentos, Campinas, v. 25, n. 3, p. 425-429, 2005.
LOPES NETO, J. P.; NASCIMENTO, J. W. B.; SILVA, V. R. Efeito do tempo de armazenagem de rações avícolas no dimensionamento de silos. Engenharia Agrícola, Jaboticabal, v. 39, n. 4, p. 518-527, 2009.

MEDEIROS, M. L.; LANNES, S. C. S. Propriedades físicas de substitutos de cacau. Ciência e Tecnologia de Alimentos, Campinas, v. 30, p. 243-253, 2010. Suplemento 1.

MENDONÇA, J. C. F.; FRANCA, A. S.; OLIVEIRA, L. S. Physical characterization of non-defective and defective Arabica and Robusta coffees before and after roasting. Journal of Food Engineering, v. 92, n. 4, p. 474-479, 2009.

MILANI, A. P. Determinação das propriedades de produtos armazenados para projetos de pressões e fluxo em silos. São Carlos: USP, 1993. 285 p.

MOHSENIN, N. N. Physical properties of plant and animal materials. New York: Gordon and Breach Science Publishers, 1986. 918 p.

MWITHIGA, G.; JINDAL, V. K. Physical changes during coffee roasting in rotary conduction-type heating units. Journal of Food Process Engineering, Maiden, v. 26, n. 6, p. 543-558, 2003.

NAKA, S. Determinação das propriedades físicas dos grãos de mamona 'Guarani' visando armazenagem em silos verticais. Campinas: UNICAMP, 2010. 105 p.

NASCIMENTO, F. C. A relação entre as pressões horizontais e verticais em silos elevados: o parâmetro K. São Carlos: USP, 2008. 119 p.

PEGG, R. B.; SHAHIDI, F. Encapsulation, stabilization, and controlled release of food ingredients and bioactives. In: RAHMAN, M. S. (Ed.). Handbook of food preservation. 2. ed. Boca Raton: CRC Press, 2007. p. 509-570.

PIMENTA, T. V.; PEREIRA, R. G. F. A.; CORRÊA, J. L. G.; SILVA, J. R. Roasting processing of dry coffee cherry: influence of grain shape and temperature on physical, chemical and sensorial grain properties. Boletim do CEPPA, Curitiba, v. 27, n. 1, p. 97-106, 2009.

PITTIA, P.; DALlA ROSA, M.; LERICI, C. R. Textural changes of coffee beans as affected by roasting conditions. LWT - Food Science and Technology, Suíça, v. 34, n. 3, p. 168-175, 2001.

PODCZECK, F.; MIAH, Y. The influence of particle size and shape on the angle of internal friction and the flow factor of unlubricated and lubricated powders. International Journal of Pharmaceutics, Filadélfia, v. 144, n. 2, p. 187-194, 1996. 
ROBBERTS, T. C. Food plant engineering systems. New York: CRC Press, 2002. 268 p.

SCHMIDT, C.A. P.; MIGLIORANZA, É.; PRUDÊNCIO, S. H. Interação da torra e moagem do café na preferência do consumidor do oeste paranaense. Ciência Rural, Santa Maria, v. 38, n. 4, p. 1111-1117, 2008.

SCHUBERT, H. Food particle technology. Part I: properties of particles and particulate food systems. Journal of Food Engineering, Essex, v. 6, n. 1, p. 1-32, 1987.

SHITTU, T. A.; LAWAL, M. O. Factors affecting instant properties of powdered cocoa beverages. Food Chemistry, London, v. 100, n. 1, p. 91-98, 2007.

SILVA, F. S.; CORRÊA, P. C.; CALIL-JÚNIOR, C.; GOMES, F. C. Ângulo de repouso, atrito interno e efetivo dos grãos de café com pergaminho. Revista Brasileira de Produtos Agroindustriais, Campina Grande, v. 8, n. 1, p. 17-23, 2006.
SINGH, P. C.; SINGH, R. K.; BHAMIDIPATI, S.; SINGH, S. N.; BARONE, P. Thermophysical properties of fresh and roasted coffee powders. Journal of Food Process Engineering, Maiden, v. 20, n. 1, p. 31-50, 1997.

VARGAS-ELÍAS, G. A. Avaliação das propriedades físicas e qualidade do café em diferentes condições de torrefação. Viçosa: UFV, 2011. 116 p.

WILHELM, L. R.; SUTER, D. A.; BRUSEWITZ, G. H. Food \& process engineering technology textbook. St. Joseph, Michigan: ASAE, 2004. 299 p.

YAN, H.; BARBOSA-CANOVAS, G. V. Compression characteristics of agglomerated food powders: effect of agglomerate size and water activity. Food Science and Technology International, Filadélfia, v. 3, n. 5, p. 351359, 1997. 
